\title{
Ultra Performance Liquid Chromatography (UPLC) Further Improves Hydrogen/Deuterium Exchange Mass Spectrometry
}

\author{
Yan $\mathrm{Wu}$ and John R. Engen \\ Department of Chemistry, University of New Mexico, Albuquerque, New Mexico, USA
}

\author{
William B. Hobbins \\ Waters Corporation, Milford, Massachusetts, USA
}

\begin{abstract}
Ultra performance liquid chromatography (UPLC) employs particles smaller than $2 \mu \mathrm{m}$ in diameter to achieve superior resolution, speed, and sensitivity compared with high-performance liquid chromatography (HPLC). We have tested the suitability of UPLC for the analysis of deuterated peptides in hydrogen exchange mass spectrometry experiments. Superior resolution and sample throughput were obtained with UPLC versus HPLC. For highly deuterated model peptides, deuterium loss using UPLC was greater than the deuterium loss observed using a conventional HPLC system, primarily as a result of the injection requirements of the UPLC system. Partially deuterated cytochrome $c$ peptides also lost more deuterium in UPLC versus HPLC, although the effect was not as pronounced as it was for the highly deuterated model peptides. The exceptional chromatographic aspects of UPLC make it a very attractive alternative to HPLC for hydrogen exchange mass spectrometry experiments. (J Am Soc Mass Spectrom 2006, 17, 163-167) ( 2006 American Society for Mass Spectrometry
\end{abstract}

$\mathrm{H}$ ydrogen exchange (HX) mass spectrometry (MS) is exceedingly valuable and has grown in popularity over recent years [1]. A typical experiment involves deuterating a protein of interest, quenching the exchange reaction by $\mathrm{pH}$ and temperature reduction, and analyzing the level and location of the deuterium with mass spectrometry [1,2]. MS analyses with electrospray ionization require a liquid chromatography (LC) step before mass analysis to remove buffer salts, and to separate the peptic peptides generated during digestion of the labeled protein(s). In the LC step, the deuterated peptides come into contact with protiated solvents and some deuterium exchanges back to hydrogen in the so called back-exchange process [3]. To retain as much deuterium during analysis and, therefore, information about the folded state of the protein when it was labeled, back-exchange must be minimized as much as possible.

Two main requirements for minimizing back-exchange are maintaining low-temperature $\left(0^{\circ} \mathrm{C}\right.$, ice bath) and completing the chromatography as rapidly as possible. The half-life of deuterium back-exchange at peptide amide linkages is between 30 to $120 \mathrm{~min}$ under

Published online January 10, 2006

Address reprint requests to Dr. J. R. Engen, Department of Chemistry, University of New Mexico, Clark Hall 242, MSC03-2060, Albuquerque, NM 87131-0001, USA. E-mail: engen@unm.edu quench conditions of $\mathrm{pH} 2.5$ and $0{ }^{\circ} \mathrm{C}[4,5]$. LC separation, therefore, must be fast. In an optimized HX MS experiment, separation may occur in as little as 5 $\min$ at $0{ }^{\circ} \mathrm{C}$ with a $\mathrm{C}-18$ reversed-phase microbore column. Such a separation is not ideal in terms of LC resolution but resolution is compromised to maintain high levels of deuterium recovery.

Ultra performance liquid chromatography (UPLC) makes it possible to perform very high-resolution separations in short periods of time [6-8] and, therefore, seems ideally suited for HX MS. UPLC utilizes solidphase particles of $1.7 \mu \mathrm{m}$ diameter to achieve superior theoretical plates and resolution. The small particles create operating pressures that are very high (in the range of 6000 to 15,000 psi). A special LC system capable of handling such high pressures recently became commercially available. We tested this UPLC system for its applicability to HX MS.

\section{Experimental}

\section{Chemicals}

Horse heart cytochrome $c$, bradykinin, methionine enkephalin, angiotensin I, porcine pepsin (1:60,000 grade), trifluoroacetic acid (TFA), and formic acid were obtained from Sigma Chemical Co. (St. Louis, MO) and used without further purification. 
Table 1. Comparison of UPLC and HPLC for HX MS

\begin{tabular}{|c|c|c|c|c|c|}
\hline System & Gradient $^{a}$ & $\begin{array}{l}\text { Flow rate } \\
(\mu \mathrm{L} / \mathrm{min})\end{array}$ & $\begin{array}{c}\text { Elution } \\
\text { time }(\min )^{b}\end{array}$ & $\begin{array}{c}\text { Separation } \\
\text { quality }\end{array}$ & $\begin{array}{l}\text { Samples } \\
\text { per hour }\end{array}$ \\
\hline UPLC & A & 100 & 2.7 & +++++ & $8-10$ \\
\hline HPLC & B & 50 & 6.0 & +++ & $4-5$ \\
\hline HPLC & B & 100 & 4.6 & ++ & $5-7$ \\
\hline HPLC & $A$ & 100 & 2.7 & + & $7-9$ \\
\hline
\end{tabular}

${ }^{a}$ Gradient A: $2 \%$ ACN for 0.7 mins, $2-15 \%$ ACN in $0.1 \mathrm{~min}, 15-90 \%$ ACN in 3.2 min; Gradient B: $5 \%$ ACN for 1 min, $5-15 \%$ ACN in 0.5 min, $15-50 \%$ ACN in $5.5 \mathrm{~min}$.

bElution time is defined here as the time that elapsed from the start of the LC gradient until the last cytochrome $c$ peptide was detected by the mass spectrometer. Elution time does not include the 2 min pepsin digest time.

${ }^{\mathrm{c}} \mathrm{A}$ typical range has been indicated because sample throughput may depend on column reconditioning, which may be a function of the specific column and sample.

\section{UPLC and HPLC}

UPLC separation was carried out with a Waters ACQUITY UPLC system. The autosampler chamber/block was set at $5{ }^{\circ} \mathrm{C}$. Due to the nature of the UPLC plumbing, the tubing between the sample loop (in the autosampler compartment) and the column was not cooled to $0{ }^{\circ} \mathrm{C}$ but the column and all tubing beyond it were immersed in an ice bath. Both mobile phase bottles were placed on ice and both mobile phases contained $0.05 \%$ TFA. The optimal UPLC elution gradient (gradient A, Table 1) was performed at a flow rate of 100 $\mu \mathrm{L} / \mathrm{min}$. The same gradient was also used at $50 \mu \mathrm{L} /$ $\mathrm{min}$ and $130 \mu \mathrm{L} / \mathrm{min}$. The system was operated in full-loop mode with a $20 \mu \mathrm{L}$ loop. The UPLC column was a $1.0 \times 50.0 \mathrm{~mm}$ ACQUITY UPLC C18 BEH (Waters) containing $1.7 \mu \mathrm{m}$ particles. The back pressure at $0{ }^{\circ} \mathrm{C}$ and 50,100 , or $130 \mu \mathrm{L} / \mathrm{min}$ averaged 4400,8200 , or 10,250 psi, respectively. HPLC separation was carried out with a Shimadzu 10AD-VP HPLC (Columbia, MD). The injector (Rheodyne 7725, Rahnert Park, CA), tubing, and column were immersed in an ice bath to minimize deuterium back-exchange. Both mobile phase bottles were placed on ice to cool the solvents and 0.05\% TFA was added to both solvents. The optimal HPLC gradient (gradient B, Table 1) was performed at a flow rate of $50 \mu \mathrm{L} / \mathrm{min}$. Other nonoptimal gradients and flow rates were also used with the HPLC (see Table 1). The HPLC column was a Jupiter Proteo, $1.0 \times 50.0 \mathrm{~mm}$, $4 \mu \mathrm{m}$ particle size, $90 \AA$ pore size (Phenomenex, Torrance, $\mathrm{CA}$ ) and produced an average back pressure of 800 to 900 psi at $0{ }^{\circ} \mathrm{C}$ and $50 \mu \mathrm{L} / \mathrm{min}$.

\section{Hydrogen Exchange and Digestion}

Highly deuterated bradykinin, methionine enkephalin, and angiotensin I were prepared by dissolving an equal molar ratio mixture of the peptides in $\mathrm{H}_{2} \mathrm{O}(\mathrm{pH} 5)$ at a concentration of $320 \mathrm{pmol} / \mu \mathrm{L}$. The mixture was diluted 15-fold with labeling buffer $\left(\mathrm{D}_{2} \mathrm{O}, 20 \mathrm{mM}\right.$ potassium phosphate, $\mathrm{pD} 7.6$ ) and maintained at $37{ }^{\circ} \mathrm{C}$ for $6 \mathrm{~h}$ (although total deuteration should occur in less than $10 \mathrm{~s}$ under these conditions [4]). The labeling reaction was quenched as described previously [9]. To prepare deuterated cytochrome $c$, the protein $(320 \mathrm{pmol} / \mu \mathrm{L})$ was diluting 15 -fold with labeling buffer. After 5 min or $22 \mathrm{~h}$ of labeling, an aliquot of $53 \mu \mathrm{L}$ was removed and deuterium exchange was quenched [9]. For peptic digestion, porcine pepsin $(2 \mu \mathrm{g} / \mu \mathrm{L})$ was added to each quenched cytochrome $c$ sample $(106 \mu \mathrm{L})$ and the mixture was incubated at room-temperature for $2 \mathrm{~min}$ before injection $(20 \mu \mathrm{L} ; 200 \mathrm{pmol})$.

\section{Mass Analysis}

Mass spectrometry was carried out with a QTOF2 (Waters) equipped with a standard ESI source. All mass spectral measurements were taken at: capillary voltage $2.7 \mathrm{kV}$, cone voltage $30 \mathrm{~V}$, source temperature $85^{\circ} \mathrm{C}$, desolvation temperature $175{ }^{\circ} \mathrm{C}$, desolvation gas 500 L/h. Each $0.5 \mathrm{~s}$ scan spanned $\mathrm{m} / \mathrm{z}$ 200-1990 with an interscan delay time of $0.1 \mathrm{~s}$. Myoglobin was infused at the end of each run for mass calibration.

\section{Data Processing and Calculations}

The measured deuterium level was obtained as described [9]. The percent measured deuterium loss during analysis was obtained by dividing the measured deuterium level by the total number of backbone amide hydrogens and subtracting the result from one. The theoretical deuterium loss of highly deuterated peptides during injection and LC was determined by calculating (with HXPep, available from Zhongqi Zhang, Amgen, Thousand Oaks, CA) the quantity of deuterium that should remain in each peptide under the $\mathrm{pH}$, temperature, and time parameters of each step. The theoretical loss during mass analysis at the MS conditions described above was estimated at 5\%, the maximum amount of loss seen in separate infusion experiments of highly deuterated peptides.

\section{Results and Discussion}

\section{Comparison of Separation}

To compare the chromatographic separation of UPLC versus HPLC for HX MS samples, two identical peptic digests of deuterated cytochrome $c$ were prepared. One was analyzed by the UPLC system and the other one by 
(a)

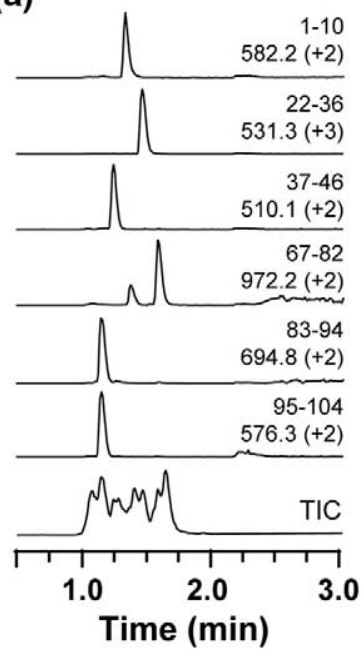

(c)

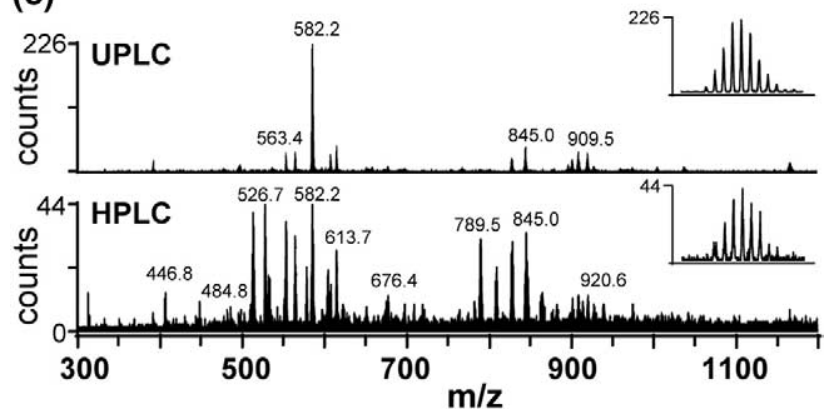

Figure 1. Comparison of separation in UPLC versus HPLC. Optimal separations corresponding to the conditions in the first two lines of Table 1 are shown for (a) UPLC and (b) HPLC. The top six traces are selected ion chromatograms of cytochrome $c$ peptides and the bottom trace is the total ion chromatogram (TIC) for the sample. The time axis indicates the time that had elapsed after sample injection and does not include 1 (UPLC) or 2 (HPLC) min delay before MS acquisition to allow the salt peak to elute. (c) Representative mass spectra of peptide 1-10 ( $\mathrm{m} / \mathrm{z}$ 582.2) comparing the purity and signal intensity in UPLC versus HPLC. The inset shows a magnification of the region around the 582.2 ion.

the HPLC system. The selected ion chromatograms of six peptic peptides were obtained and are shown in Figure 1. As the two separation columns were not identical (see Experimental section for details of the columns), the separation efficiency was only compared qualitatively. Peptides in UPLC eluted within a 0.75 min window (Figure 1a) and all of the peptides were highly resolved. In contrast, the optimal HPLC separation (Figure $1 b$ ) took longer with much poorer quality. While HPLC separation speed could be improved with various gradients (Table 1), the separation quality declined substantially when the nonideal flow rate and nonideal gradient were used in HPLC.

As shown in Table 1, the clear advantage of using UPLC instead of HPLC for hydrogen exchange mass spectrometry is the sample throughput potential and the quality of the separation. While we tested the system with a relatively small protein (cytochrome $c, 12$ $\mathrm{kD}$ ) one would expect that the ability to resolve hun- dreds of peptic peptides from digestion of much larger proteins would be a clear advantage of UPLC. Because the peaks are so sharp in UPLC, the MS signal for peptic peptides also improves with UPLC analysis (Figure 1c).

\section{Deuterium Loss in Model Peptides}

The deuterium loss in UPLC was compared with that observed using HPLC by first monitoring deuterium loss in three highly deuterated peptides. The peptides were labeled with $\mathrm{D}_{2} \mathrm{O}$ and the exchange quenched just before sample injection, thereby washing away deuterium at side-chain positions which exchange very rapidly [3]. The analysis can be divided into three steps: injection, chromatography, and mass analysis. As a result of the UPLC sample injection requirements, these three steps were slightly different for HPLC and UPLC.

The injection in HPLC analysis was done manually and took maximally $3 \mathrm{~s}$ with an injector submerged in an ice bath. Deuterium loss during HPLC injection, therefore, should theoretically be almost zero (solid black area of theory bars, Figure 2). For UPLC analysis, a standard AQUITY UPLC system was used, and injection proceeded via an autosampler. Samples were placed in the autosampler block immediately after being quenched. The autosampler sample block and chamber were maintained at $5{ }^{\circ} \mathrm{C}$ and the time that elapsed from the moment the autosampler needle touched the sample until the sample in the loop was placed into the solvent flow-stream was $\sim 50 \mathrm{~s}$. The time spent between the sample loop and the column was $\sim 12$ s. The UPLC injector, sample loop, and tubing precolumn were not cooled with an ice bath as these parts are buried within the housing of the standard AQUITY instrument. To partially compensate, the solvent bottles were placed on ice. Based on our experience, the temperature of the solvents and the sample loop during the UPLC injection process was estimated to be $12{ }^{\circ} \mathrm{C}$. In theory, therefore, UPLC samples were

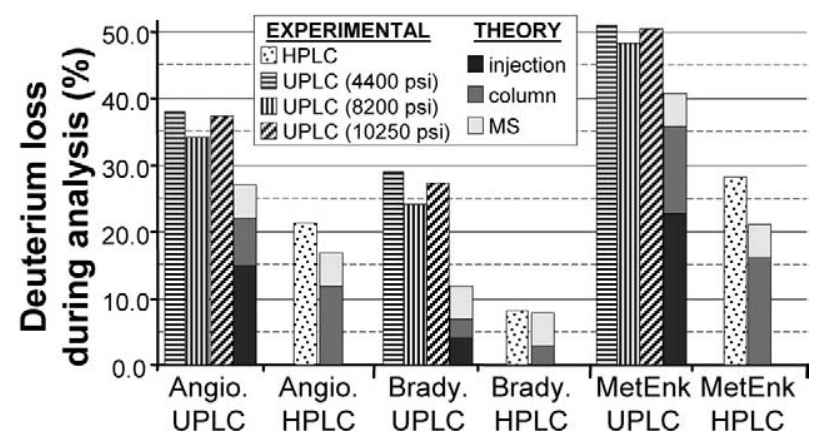

Figure 2. Comparison of deuterium losses in highly deuterated peptides angiotensin I (angio.), bradykinin (brady.) and methionine enkephalin (MetEnk). Measured losses are shown with patterned bars and theoretical losses (see Experimental section for details of the calculation) are shown as solid bars. The HPLC flow rate was $50 \mu \mathrm{L} / \mathrm{min}(800-990 \mathrm{psi})$ while the UPLC flow rate was either $50 \mu \mathrm{L} / \mathrm{min}(\sim 4400 \mathrm{psi}), 100 \mu \mathrm{L} / \mathrm{min}(\sim 8200 \mathrm{psi})$ or 130 $\mu \mathrm{L} / \mathrm{min}(\sim 10,250 \mathrm{psi})$. 
exposed to $12{ }^{\circ} \mathrm{C}, \mathrm{pH} \sim 2.0$ for about 1 min during injection. Such conditions should cause deuterium loss during the UPLC injection cycle ranging from 4 to $23 \%$, depending on the sequence (solid black area of theory bars, Figure 2).

Unlike the analysis of cytochrome $c$ peptides above (Figure 1), the chromatography step for highly deuterated peptides was the same for UPLC and HPLC (Table 1 , gradient $\mathrm{A}, 100 \mu \mathrm{L} / \mathrm{min}$ ). Deuterated peptides were exposed to $0{ }^{\circ} \mathrm{C}, \mathrm{pH} \sim 2.0$ for 2.5 min during chromatography and theoretical deuterium losses during HPLC were calculated using these conditions (solid gray area of theory bars, Figure 2). Theoretical UPLC losses during LC used the same conditions but compensated for the fact that the samples in UPLC had already been exposed to injection conditions for $1 \mathrm{~min}$. Theoretical losses for the MS step of UPLC and HPLC were assumed to be $5 \%$ (light-gray part of theory bars, Figure 2).

Based entirely on theoretical calculations of deuterium loss due to back-exchange during the various steps of analysis, one would expect the deuterium losses in UPLC to be between 4 to $20 \%(\bar{X}=12 \%)$ higher than the losses in HPLC (compare theory bars, Figure 2 ), primarily as a result of the difference in the injection and chromatography steps. The experimental results of analysis of highly deuterated peptides with UPLC indicated an apparent increase in deuterium loss of 13 to $20 \%(\bar{X}=16.5 \%)$ over that obtained with HPLC analysis (compare stripped bars with speckled bars, Figure 2). Although pressure significantly alters HX rates in folded proteins, changes are relatively insignificant in unstructured proteins and peptides [10]. Although we observed slight differences between deuterium losses in the unstructured, highly deuterated peptides tested here, higher pressure did not correlate with higher deuterium loss (Figure 2). We conclude that the injection step in UPLC accounts for much of the additional deuterium losses that were observed for highly deuterated peptides.

\section{Analysis of Deuterated Cytochrome c Peptides}

To compare UPLC and HPLC deuterium recovery in a more typical hydrogen exchange sample, cytochrome $c$ was deuterated for various times and digested offline with pepsin before UPLC or HPLC analysis. Figure 3 shows results for $5 \mathrm{~min}$ and $22 \mathrm{~h} \mathrm{D}_{2} \mathrm{O}$ incubations. The deuterium levels of six representative cytochrome $c$ peptides (same as in Figure 1) illustrate that in general, UPLC deuterium recovery was less than that of HPLC, again likely as a result of the injection system in the UPLC. It should be noted that in Figure 2 we have plotted deuterium loss \% whereas in Figure 3 we have plotted relative deuterium level \%. This was done because cytochrome $c$ is folded during labeling and is only partially deuterated at both time points. We cannot determine the loss \%, as the amount of deuterium present upon quenching was a function of cytochrome

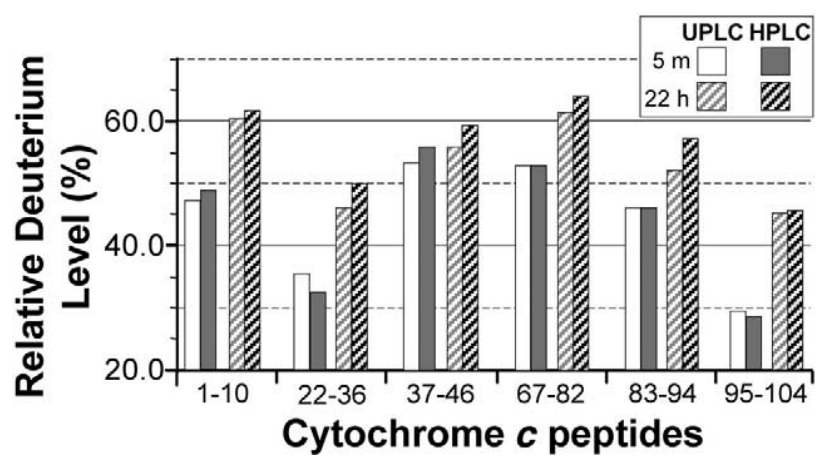

Figure 3. Deuterium level of peptides from a digestion of partially deuterated cytochrome $c$. Cytochrome $c$ was incubated in $99 \% \mathrm{D}_{2} \mathrm{O}$ pD 7.6 for either $5 \mathrm{~min}$ or $22 \mathrm{~h}$, exchange was quenched, and the samples were digested and analyzed as described in the Experimental section. The residue numbers of each peptide are shown. As no adjustment has been made for back-exchange during analysis, the deuterium level is relative.

$c$ structure and was unknown. Therefore, the differences in deuterium levels for cytochrome $c$ peptides cannot be directly compared with differences seen in highly deuterated peptides.

In general, deuterium losses in cytochrome $c$ peptides were not as severe as was demonstrated for highly deuterated peptides. We hypothesize that the reasons for this are 2-fold. First, because the typical deuterium levels of the cytochrome $c$ peptides in Figure 3 were between 35 to $60 \%$ (compared with near 100\% deuteration of the peptides in Figure 2), the back-exchange during the chromatography step, where the concentration of $\mathrm{H}_{2} \mathrm{O}$ is effectively $100 \%$, should be less for these partially deuterated peptides than it would be for $100 \%$ deuterated peptides (explained in detail in the supplement to [3] and in Appendix II of [11]. Second, the cytochrome $c$ experiments contained a digestion step that was not present in the analysis of highly deuterated peptides in Figure 2. The digestion step is where back-exchange of those amide hydrogens that exchange most rapidly at the quench $\mathrm{pH}$ would have occurred. Rapidly exchanging amide hydrogens would have been equally lost in both UPLC and HPLC analyses since both had identical digestion steps. Modeling the expected back-exchange of deuterated versions of the cytochrome $c$ peptides (data not shown) shows that the expected deuterium levels in the peptides should be very similar in UPLC versus HPLC even with the additional losses anticipated in the injection cycle of the UPLC system. Indeed, the observed differences in deuterium levels of cytochrome $c$ peptides in UPLC versus HPLC were relatively minor.

The results shown in Figure 3 are far more typical of a sample that would actually be analyzed with a UPLC system. We estimate that losses for samples such as this with UPLC would be around 5\% higher than analysis of the same samples with traditional HPLC. Such a modest difference seems tolerable in light of the other tremendous chromatographic advantages provided by 
UPLC. We anticipate that modifications to the standard Waters UPLC sample introduction system used here (including more and/or better cooling of the sample chamber, loop, and associated precolumn tubing) would reduce some of the additional back-exchange that occurs in the standard injection system. It remains to be seen how online pepsin digestion will be integrated into a UPLC system.

\section{Acknowledgments}

The authors are pleased to acknowledge financial support from the NIH (P20-RR016480 and R01-GM070590), technical assistance from S. Griffin, and critical reading of the manuscript by T. E. Wales.

\section{References}

1. Wales, T. E.; Engen, J. R. Hydrogen exchange mass spectrometry for the analysis of protein dynamics. Mass Spectrom. Rev. 2005, in press.
2. Hoofnagle, A. N.; Resing, K. A.; Ahn, N. G. Protein analysis by hydrogen exchange mass spectrometry. Annu. Rev. Biophys. Biomol. Struct. 2003, 32, 1-25.

3. Zhang, Z.; Smith, D. L. Determination of amide hydrogen exchange by mass spectrometry: A new tool for protein structure elucidation. Protein Sci. 1993, 2, 522-531.

4. Bai, Y.; Milne, J. S.; Mayne, L.; Englander, S. W. Primary structure effects on peptide group hydrogen exchange. Proteins: Struct. Funct. Genet. 1993, 17, 75-86.

5. Englander, J. J.; Rogero, J. R.; Englander, S. W. Protein hydrogen exchange studied by the fragment separation method. Anal. Biochem. 1985, 147, 234-244.

6. Swartz, M. E. UPLC: An introduction and review. J. Liq. Chromatogr. 2005, 28, 1253-1263.

7. Swartz, M. E. Ultra performance liquid chromatography (UPLC): An introduction. LC GC 2005, 23, 8-14.

8. Plumb, R.; Castro-Perez, J.; Granger, J.; Beattie, I.; Joncour, K.; Wright, A. Ultra-performance liquid chromatography coupled to quadrupoleorthogonal time-of-flight mass spectrometry. Rapid Commun. Mass Spectrom. 2004, 18, 2331-2337.

9. Engen, J. R.; Smith, D. L. Investigating the higher order structure of proteins: Hydrogen exchange, proteolytic fragmentation and mass spectrometry. Methods Mol. Biol. 2000, 146, 95-112.

10. Carter, J. V.; Knox, D. G.; Rosenberg, A. Pressure effects on folded proteins in solution. Hydrogen exchange at elevated pressures. J. Biol. Chem. 1978, 253, 1947-1953.

11. Zhang, Z. Protein hydrogen exchange determined by mass spectrometry: A new tool for probing protein high-order structure and structural changes. Ph.D. Thesis, Purdue University, 1995; pp 1-223. 